\section{NOVEL MICROBIAL IMMUNOTHERAPY APPROACH FOR THE TREATMENT OF BLADDER CANCER}

${ }^{1}$ Nicholas Glanville, ${ }^{1}$ Tobi Oke, ${ }^{2}$ Sonia Domingos-Pereira, ${ }^{2}$ Lenka Polak, ${ }^{2}$ Denise NardelliHaefliger, 'Livija Deban*. ${ }^{1}$ Prokarium Ltd, London, UK; ${ }^{2}$ Centre Hospitalier Universitaire Vaudois, Lausanne, Switzerland

Background Microbial immunotherapy, in the form of intravesical Bacillus Calmette-Guérin (BCG), is the standard-of-care for non-muscle invasive bladder cancer (NMIBC). BCG therapy is associated with significant side-effects, high disease recurrence and progression rates, and product supply shortages, leaving a significant unmet medical need for bladder cancer patients. ${ }^{1}{ }^{2}$ We sought to establish the preclinical safety and efficacy of live-attenuated Salmonella enterica Typhi strain ZH9 ( $\Delta$ aroC, $\Delta$ ssaV) as a novel microbial immunotherapy.

Methods Therapeutic efficacy of intravesical ZH9 was established in the murine orthotopic, syngeneic MB49 bladder tumor model. Tumor-bearing animals were treated with a single intravesical dose of ZH9 or OncoTice BCG and long-term survival comparisons were evaluated by log-rank (Mantel-Cox) test. ZH9 interaction with urothelial cancer cells was investigated using in vitro invasion assays and flow cytometry staining for intracellular Salmonella common antigen (CSA-1) and propidium iodide for cell death. Local immune responses were analyzed by flow cytometry staining of disaggregated mouse bladders.

Results Mice treated with a single intravesical dose of ZH9 2 days after MB49 tumor inoculation demonstrated significant survival benefit compared to vehicle treated (median survival 49.5 vs. 31 days, $\mathrm{p}=0.003$ ) and $\mathrm{BCG}$ treated animals (median survival 49.5 vs. 27.5 days, $\mathrm{p}<0.001$ ). A second, stringent model setup with intravesical treatment 4 days after tumor inoculation showed significant efficacy of ZH9 versus vehicle and BCG (median survival 30 vs. $20.5(\mathrm{p}=0.003)$ and 23.5 $(\mathrm{p}=0.025)$ days, respectively). Surviving ZH9 treated animals demonstrated $100 \%$ protection from tumor take following repeated intravesical challenge with MB49 tumor cells, suggesting lasting anti-tumor immunity resulting from $\mathrm{ZH} 9$ treatment. In vitro, intracellular flow cytometry in human (UMUC3, T24, RT4, 5637) and mouse (MB49) urothelial cancer cell lines showed that ZH9 invaded and induced cell death in all cell lines. In vivo, a single treatment with intravesical $\mathrm{ZH} 9$ resulted in strong cellular immune responses characterized by recruitment of monocytes, NK cells, CD4+ and $\mathrm{CD} 8+\mathrm{T}$ cells, and dendritic cells with an activated, cross-presenting (Ly6C+, CD103+) phenotype. Intravesical ZH9 resulted in a greater magnitude and duration of immune cell recruitment in the urothelium compared to a single equivalent dose of intravesical BCG.

Conclusions Live-attenuated Salmonella strain ZH9 demonstrated a significant survival benefit over the standard-of-care OncoTice BCG in an orthotopic bladder cancer model. ZH9 demonstrated direct tumour cell killing in vitro and induction of robust cellular immune responses in vivo. Preclinical studies indicate significant therapeutic potential of intravesical ZH9 as a novel microbial immunotherapy in bladder cancer.

\section{REFERENCES}

1. Liu Y, Lu J, Huang Y, Ma L. Clinical spectrum of complications induced by intravesical immunotherapy of Bacillus Calmette-Guérin for bladder cancer. J Oncol 2019 March 10;2019:6230409.

2. vanRhijn BW, Burger $M$, Lotan $Y$, et al. Recurrence and progression of disease in non-muscle-invasive bladder cancer: from epidemiology to treatment strategy. Eur Urol 2009 September;56(3):430-42.
Ethics Approval The studies were conducted under approval from Pennsylvania State College of Medicine Institutional Animal Care and Use Committee approval number 47682, or with approval of the Cantonal Veterinary Office of Canton de Vaud, Switzerland.

http://dx.doi.org/10.1136/jitc-2021-SITC2021.878 